\title{
ESTUDIO EXPLORATORIO DE RELACIÓN ENTRE LA PERCEPCIÓN DE APOYO SOCIAL INSTRUMENTAL Y LA MODIFICACIÓN DE ROLES FAMILIARES EN MUJERES CON CÁNCER DE MAMA
}

\author{
AN EXPLORATORY STUDY ABOUT INSTRUMENTAL SOCIAL SUPPORT PERCEPTION \\ AND FAMILIAR ROLE MODIFICATION IN WOMAN WITH BREAST CANCER
}

Leivy Patricia González-Ramírez' , Cristina Estrada-Pineda², Rebeca Robles-García ${ }^{3}$, Imelda Orozco-Mares ${ }^{4}$, Rosa Martha Meda-Lara ${ }^{5}$ y Adrian Daneri-Navarro 6

${ }^{1}$ Centro Universitario de Ciencias de la Salud, Universidad de Guadalajara, México

${ }^{2}$ Centro Universitario de Ciencias Sociales y Humanidades, Universidad de Guadalajara, México

${ }^{3}$ Dirección de Investigaciones Epidemiológicas y Psicosociales, Instituto Nacional de Psiquiatría Ramón de la Fuente Muñiz, México

${ }^{4}$ Departamento de Ciencias de las Salud del Centro Universitario de Tonalá, Universidad de Guadalajara, México

${ }^{5}$ Departamento de Psicología Básica, Centro Universitario de Ciencias de la Salud, Universidad de Guadalajara, México

- Departamento de Fisiología, Centro Universitario de Ciencias de la Salud, Universidad de Guadalajara, México

Resumen

Objetivo: Explorar la relación entre las fuentes y el tipo de apoyo instrumental brindado a las mujeres con Cáncer de Mama (CM) con la modificación de roles y actividades cotidianas en las pacientes y sus allegados.

Método: Se realizó un estudio cualitativo centrado en la etnografía de 9 pacientes con CM. A través del software MAXQDA v10, se hizo un análisis temático del discurso para posteriormente elaborar tablas cruzadas para el análisis de las variables en estudio.

Resultados: Para estas mujeres, la familia nuclear constituye la principal fuente de apoyo instrumental, al mismo tiempo que son quienes suelen modificar más sus actividades cotidianas y roles sociales. Las mujeres con CM expresan dificultad para delegar principalmente actividades del hogar debido a 3 razones: la responsabilidad de madre, la sensación de utilidad y una ideología tradicional de género.
Abstract

Objective: To explore the relationship between the sources and types of instrumental support provided to women with breast zancer, with changes in roles and daily activities in patients and their relatives.

Methods: Qualitative study focused on the ethnography of 9 patients with nreast cancer. Through MAXQDA v10 software, we did a thematic analysis of discourse and developed cross tables for the analysis of the study variables.

Results: For these women, the nuclear family is the main source of instrumental support, also are who often modify their daily activities and social roles. Women with breast cancer expressed difficulty delegating household chores, principally due to three reasons: Her mother's responsibility, a sense of usefulness and traditional gender ideology.

Conclusions: The impact of cancer in women has unique consequences, because they

\section{Correspondencia:}

Mtra. Leivy Patricia González Ramírez

Centro Universitario de Ciencias de la Salud. Universidad de Guadalajara

Sierra Mojada 950, Edificio P, piso 1, Coordinación de Investigación. CP 44350 - Guadalajara, México

E-mail: leivypagora@yahoo.com.mx 
Conclusiones: El impacto del cáncer en la mujer tiene consecuencias únicas, debido al papel central que ellas asumen en la vida familiar. Los movimientos funcionales, afectivos y relacionales que ocurren en la familia ante un diagnóstico de CM parece ser un hecho frecuente $y$ de gran trascendencia, que ha sido poco estudiado en nuestra población. Estos aspectos deben ser considerados como un elemento importante en la comprensión y el soporte que se debe otorgar a las mujeres con $\mathrm{CM}$ y a sus familias.

Palabras clave: Cáncer de mama, apoyo social, roles sociales. assume central role in family life. Functional, affective and relational movements occurring in the family with breast cancer diagnosis, seems to be frequent and with important implications, which has been little studied in our population. These aspects should be considered as an important element in the understanding and support given to Breast Cancer patients and their families.

Keywords: Breast cancer, social support, social roles.

\section{INTRODUCCIÓN}

El cáncer de mama $(\mathrm{CM})$ es un problema de salud a escala mundial, que figura como la principal causa de muerte en mujeres por tumores malignos ${ }^{(1-3)}$. En México se informa que entre 1955 y 1960, la tasa era alrededor de 2 a 4 muertes por 100.000 mujeres, elevándose de manera sostenida hasta alcanzar una cifra cercana a 9 por 100.000 para la mitad de la década de $1990^{(4)}$, para el 2006 la tasa de mortalidad ya era de 15,8 por cada 100.000 habitantes ${ }^{(5)}$. En 2008, Jalisco alcanzó una tasa de incidencia de 17,97 por 100.000 habitantes, duplicándose por sexo a 35,18 por 100,000 mujeres ${ }^{06}$.

El CM impacta en muchas esferas de la vida de las pacientes. En muchos casos, los efectos secundarios de algunos tratamientos, tornan imposible enfrentar de modo adecuado y productivo su vida cotidiana ${ }^{(7)}$. Se ha documentado por ejemplo que el linfedema, el dolor, la depresión, la fatiga o el Ilamado chemobrain (deterioro cognitivo que se manifiesta con pérdida de memoria a corto plazo, atención y problemas de concentración), pueden repercutir en la funcionalidad de la paciente de manera permanente o prolongada ${ }^{(8-11)}$. De hecho, la pérdida de trabajo constituye sin duda una consecuencia adversa potencial de cáncer ${ }^{(12-13)}$; y se ha reportado que, las mujeres que reciben quimioterapia experimentan muchos efectos secundarios como náuseas y fatiga, y se asocia con deficiencias en los resultados dentro del trabajo o a un abandono voluntario del empleo ${ }^{(12)}$.

Respecto a las relaciones afectivas más cercanas, con familiares y amigos, se puede observar un impacto significativo en los roles preestablecidos en la vida cotidiana, por lo que en la actualidad se insiste en la necesidad de incorporar una visión integral al estudio e intervención de las personas que padecen cáncer ${ }^{(14)}$. En esta dirección, la psicooncología entiende a la persona con una enfermedad como un ser biopsicosocial, e intenta procurar apoyo tanto al paciente como a los familiares directos $^{(15)}$. Se conoce que al no ser manejados todos los aspectos y los miembros de la familia de manera integral por el equipo básico de salud, será mayor el deterioro de la calidad de vida de la paciente ${ }^{(16)}$.

Entendiendo la dinámica y trascendencia de la relaciones, el apoyo social, tanto formal (de instituciones hospitalarias y asistenciales) como informal (de familia y comunidad), resulta uno de los aspec- 
tos a considerar en esta problemática, sea por los aspectos benéficos que conlleva su presencia, como de sus implicaciones negativas en el transcurso de la intervención y en la evolución de la enfermedad que se relacionan con su ausencia ${ }^{(17-19)}$. El apoyo social consiste en el soporte emocional, asesoramiento $u$ orientación, así como en la ayuda material y de servicios que las personas obtienen de la red social a la que pertenecen $^{(20)}$.

Entre las fuentes de apoyo informal destaca la pareja, sobre todo en la fase del diagnóstico ${ }^{(19,21-23)}$. El apoyo brindado puede ser emocional, informativo e instrumental. El emocional incluye demostraciones de empatía, consuelo, confianza, amor y cariño. El informativo, consiste tanto en brindar información, como en ayudar a acceder a esta o a comprenderla. El instrumental puede ser otorgado con recursos económicos, cuidado de los niños o llevando a cabo labores del hogar, e incluso apoyando en la toma de decisiones ${ }^{(23-24)}$. Los tipos de apoyo más frecuentes en mujeres con $\mathrm{CM}$ son el emocional y el instrumental ${ }^{(25)}$.

El objetivo del presente trabajo fue explorar la relación entre las fuentes y el tipo de apoyo instrumental brindado a las mujeres con $\mathrm{CM}$, con la modificación de roles y actividades cotidianas en las pacientes y sus allegados.

\section{MÉTODO}

Se realizó un estudio cualitativo centrado en la etnografía, ya que buscó un entendimiento globalizado y no segmentado de la realidad. La investigación etnográfica parte del planteamiento de un problema preliminar, en este caso el CM, posteriormente transforma los problemas preliminares en un cuerpo de cuestiones a partir de las cuales se puedan extraer respuestas teóricas. La etnografía admite las experiencias subjetivas tanto del investigador como de los participantes, ofreciendo así una profundidad en la comprensión de la que carecen a menudo otros enfoques ${ }^{(26)}$.

\section{Participantes}

Se incluyeron 9 mujeres con CM seleccionadas por muestreo de selección de estructuras. Se facilitó el contacto con las participantes a través del Coordinador Nacional de la United States Latin America Cancer Research Network, que cuenta con convenios locales con distintos hospitales para la realización de proyectos de investigación en cáncer. Todas las pacientes fueron mayores de edad y habían sido diagnosticadas con $\mathrm{CM}$ en un periodo no mayor a un año. Todas las participantes firmaron una carta de consentimiento informado donde se aclaró que la información que proporcionarían sólo sería utilizada con fines de investigación y sería estrictamente confidencial.

Las características sociodemográficas de las pacientes se muestran en la tabla 1.

\section{Recolección de datos}

Se usaron diversas técnicas que favorecieron la comprensión de la población de estudio. La observación participante y no participante para obtener datos empíricos de primera mano de los fenómenos tal como se dan en los escenarios de la vida cotidiana. Estas se realizaron en las visitas a los hospitales, algunas de ellas dentro de la misma institución, o en los alrededores.

Una libreta de campo en donde se registraron detalles sobre el escenario y la situación del encuentro con cada una de las pacientes entrevistadas. Así mismo, se añadieron percepciones sobre emociones o aspectos implícitos en alguna situación, pensamientos o contribuciones propias de la subjetividad del investigador. Toda esta información adicional apoyó en la interpretación de datos y la elaboración de conclusiones. 


\begin{tabular}{|c|c|c|c|c|c|c|c|c|c|}
\hline 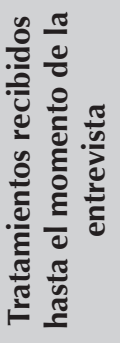 & 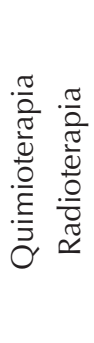 & $\begin{array}{l}\frac{\pi}{2} \\
\frac{\pi}{\frac{\pi}{0}} \\
\frac{0}{\frac{0}{E}} \\
\frac{0}{2}\end{array}$ & 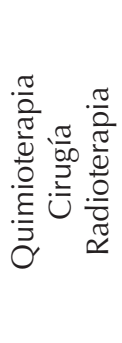 & $\begin{array}{l}\frac{\pi}{2} \\
\frac{\pi}{0} \\
\frac{0}{0} \\
\frac{0}{E} \\
\frac{0}{2} \\
0\end{array}$ & 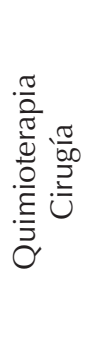 & 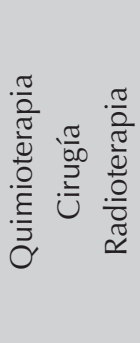 & 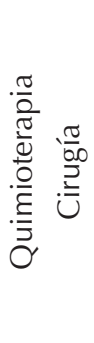 & 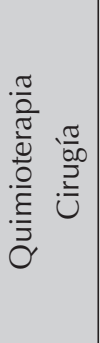 & 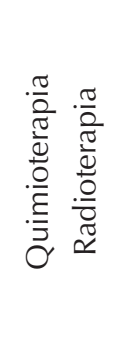 \\
\hline 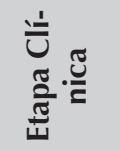 & $\leqq$ & $\cong$ & $\leqq$ & $\cong$ & $\cong$ & $\cong$ & $\cong$ & $\leqq$ & $\cong$ \\
\hline 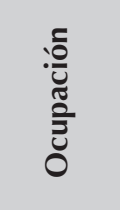 & 足 & $\frac{\frac{\pi}{2}}{\frac{0}{0}} \frac{\frac{0}{\pi}}{\frac{\pi}{0}}$ & 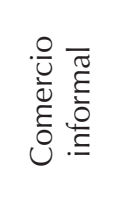 & 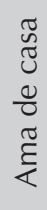 & 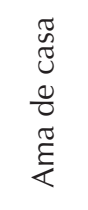 & $\begin{array}{l}\tilde{J} \\
\tilde{J} \\
0 \\
0 \\
\tilde{0} \\
\tilde{\varepsilon}\end{array}$ & 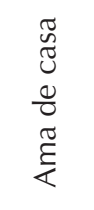 & 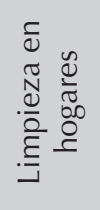 & 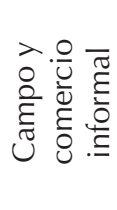 \\
\hline 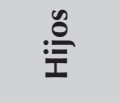 & Z & in & i & is & is & ఉ & $\bar{\omega}$ & ํ & $\bar{n}$ \\
\hline 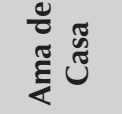 & is & is & is & is & $\bar{n}$ & in & is & Z & $\bar{n}$ \\
\hline 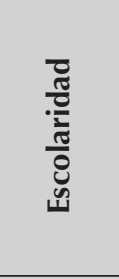 & 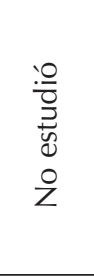 & 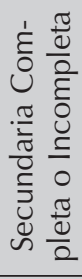 & 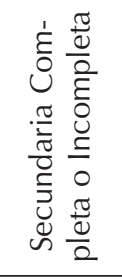 & 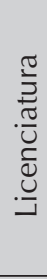 & 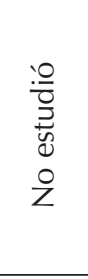 & 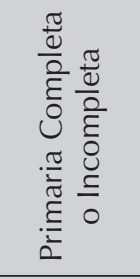 & 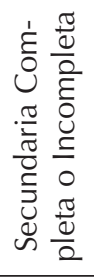 & 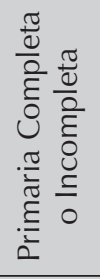 & 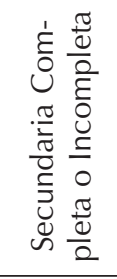 \\
\hline 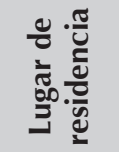 & $\begin{array}{l}\overline{\widetilde{\sigma}} \\
\stackrel{2}{\alpha}\end{array}$ & $\begin{array}{l}\overline{\widetilde{T}} \\
\stackrel{2}{\simeq}\end{array}$ & 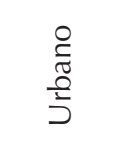 & 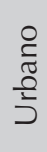 & $\begin{array}{l}\overline{\widetilde{0}} \\
\stackrel{\bar{\alpha}}{2}\end{array}$ & $\begin{array}{l}\overline{\widetilde{\pi}} \\
\stackrel{2}{\simeq}\end{array}$ & 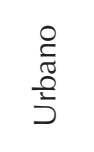 & $\begin{array}{l}\overline{\widetilde{\sigma}} \\
\stackrel{2}{\propto}\end{array}$ & $\begin{array}{l}\overline{\widetilde{\pi}} \\
\stackrel{\overline{2}}{\simeq}\end{array}$ \\
\hline$\frac{0}{\frac{0}{0}} \cdot \frac{7}{4}$ & $\begin{array}{l}\frac{\pi}{0} \\
\tilde{J} \\
\tilde{U}\end{array}$ & $\begin{array}{l}\frac{\pi}{0} \\
\tilde{N} \\
\tilde{U}\end{array}$ & $\begin{array}{l}\frac{\pi}{\tilde{J}} \\
\tilde{J} \\
\tilde{U}\end{array}$ & $\begin{array}{l}\frac{\pi}{\tilde{D}} \\
\tilde{J} \\
\tilde{U}\end{array}$ & $\begin{array}{l}\frac{\pi}{0} \\
\text { ల్ } \\
\tilde{J}\end{array}$ & $\begin{array}{l}\frac{\pi}{0} \\
\text { J } \\
\text { Ũ }\end{array}$ & $\begin{array}{l}\frac{\pi}{\tilde{D}} \\
\tilde{J} \\
\tilde{U}\end{array}$ & 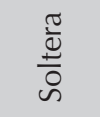 & $\begin{array}{l}\frac{\pi}{0} \\
\mathbb{J} \\
\tilde{N}\end{array}$ \\
\hline 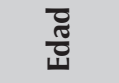 & $\stackrel{\forall}{\forall}$ & m & $\stackrel{Ð}{\forall}$ & $\stackrel{\mathrm{Ln}}{\mathrm{m}}$ & రृ & 는 & $\underset{\forall}{\forall}$ & ש & m \\
\hline 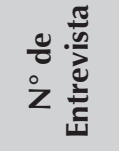 & - & $N$ & $m$ & $\nabla$ & $L \cap$ & 0 & $\wedge$ & $\infty$ & $a$ \\
\hline
\end{tabular}


Se audiograbaron entrevistas a profundidad, las cuales se realizaron cara a cara, entre el investigador y las pacientes, y se orientaron a entender las perspectivas del entrevistado sobre su vida, experiencias o situaciones personales tal y como eran expresadas por sus propias palabras ${ }^{27}$. Se elaboraron ejes temáticos de acuerdo con los objetivos del estudio. Las entrevistas no tuvieron una estructura preestablecida, sin embargo se apegaron a una guía de entrevista que señaló los puntos fundamentales de interés.

\section{ANÁLISIS DE DATOS}

Las entrevistas audiograbadas fueron transcritas a Word Office, posteriormente se exportaron a MAXQDA v10, un software profesional para el análisis de datos cualitativos. En éste se hicieron múltiples lecturas hasta la familiarización de los relatos, se continuó con un análisis temático, codificando las variables de interés e identificando núcleos temáticos, seleccionando como categorías centrales el apoyo instrumental y la modificación de roles, para derivar de ahí otras variables y establecer sus relaciones. Posteriormente se organizaron los datos utilizando tablas cruzadas, con las variables de los distintos tipos de apoyo instrumenta. Finalmente se recurrió al Visualizador de la Matriz de Códigos, para evidenciar la modificación de roles no solo en la paciente sino en otras fuentes cercanas.

Tal como sugiere Kornblit(28) la interpretación de los datos dotó de significado las inferencias acerca de las regularidades observadas, teniendo en cuenta la percepción tanto de las pacientes entrevistadas como de los investigadores, que se articularon al tiempo con el contexto socio cultural correspondiente. Las expresiones idiomáticas de las pacientes fueron respetadas en los fragmentos de las narraciones expuestas.

\section{RESULTADOS}

Las narraciones de las entrevistadas se dividen en dos apartados importantes de análisis. En primer lugar, el tipo de apoyo que las mujeres perciben recibir de diferentes fuentes, y en segundo lugar los cambios en los roles que surgieron a partir del diagnóstico tanto en ellas como en sus allegados.

Tras el análisis de las narraciones se observó que el apoyo instrumental resulta una constante en el discurso de las pacientes. En la familia de procreación fueron los esposos los que les proporcionaban con mayor frecuencia, le siguieron los hijos y la familia de origen, tanto el sistema fraterno como los padres (Ver tabla 2).

\section{Tabla 2. Fuentes de Apoyo Instrumental}

\begin{tabular}{|c|c|}
\hline $\begin{array}{c}\text { Persona que brinda } \\
\text { el apoyo }\end{array}$ & $\begin{array}{c}\text { Pacientes } \\
\text { que lo } \\
\text { refieren }\end{array}$ \\
\hline Esposo & 7 \\
\hline Hijos & 6 \\
\hline Hermanos & 6 \\
\hline Padre y/o madre & 5 \\
\hline $\begin{array}{c}\text { Familia extensa (Tíos, sobrinos, } \\
\text { yerno, cuñados) }\end{array}$ & 5 \\
\hline Instituciones / Personal de salud & 5 \\
\hline Amigos / Vecinos / Compañeras & 4 \\
\hline
\end{tabular}

El apoyo instrumental se expresó de diversas maneras, por ejemplo, el dar cosas, como dinero, comida o medicamentos; o incluso, proporcionar alojamiento temporal a la paciente y su esposo (incluso hijos), importante para aquellas mujeres que residen en área rural y que debían trasladarse a los hospitales especializados en oncología, concentrados en áreas urbanas. Frecuentemente se refirió también el acompañamiento a citas, transporte, 
preparación de comidas o remedios, y cuidados a la paciente derivados de su diagnóstico y/o tratamiento (Ver tabla 3).
Los hallazgos reflejan que todas las pacientes modificaron sus actividades COtidianas y por ende, sus roles sociales. Las

\section{Tabla 3. Narraciones sobre tipos de apoyo instrumental}

\begin{tabular}{|c|c|}
\hline Ejemplos de Narraciones & Código \\
\hline $\begin{array}{l}\text { P: ...Cómo tengo una vecina allí, ahí le dije "¡Ay te encargo a mis hijos, de darles } \\
\text { una regañada sí, si se pasan!"..., y cuando llego ellos ya... "Tú muchacho esto, } \\
\text { tus muchachas lo otro" “Sí, sí, tú!" le digo "los encarrilas, dales una buena rega- } \\
\text { ñada cuando se pasen". (Entrevista 2) }\end{array}$ & \multirow{2}{*}{$\begin{array}{l}\text { Apoyo en } \\
\text { actividades } \\
\text { del hogar y/o } \\
\text { cuidado de } \\
\text { los hijos }\end{array}$} \\
\hline $\begin{array}{l}\text { P....como orita no puedo acercarme a la estufa, ella me calienta (su hija) o, o en } \\
\text { la noche este... "¿qué vas a querer de cenar?", tengo una tía también que ahorita } \\
\text { está viviendo conmigo... ella me hace quehacer. (Entrevista } 3 \text { ) }\end{array}$ & \\
\hline $\begin{array}{l}\text { P: Mi esposo pues él trabaja y... ps se le hace pesado el gasto, pero dice él "Pues } \\
\text { primero está tu salud y ya después" dice "Dios que nos ayude de alguna manera" } \\
\text { (Entrevista 6) }\end{array}$ & \multirow{2}{*}{$\begin{array}{l}\text { Dar cosas } \\
\text { (Dinero, co- } \\
\text { mida, etc.) }\end{array}$} \\
\hline $\begin{array}{l}\text { P.... y te digo por parte de mis papás, ellos venden verdura y fruta y me mandan } \\
\text { verdura, me mandan fruta para que no me haga falta nada de eso. (Entrevista 3) }\end{array}$ & \\
\hline $\begin{array}{l}\text { P: Mmmm, no pos ellos nos han apoyado bien bonito todos, nosotros llegamos y } \\
\text { como si juera su casa de uno, lo tratan a uno. (Entrevista 1) }\end{array}$ & Alojamiento \\
\hline $\begin{array}{l}\text { P: El apoyo principalmente que he, que he sentido ha sido el de mi hermana. Te } \\
\text { di, te digo ella trabaja aquí, este... yo sé que por ella me atendieron rápido, y ella } \\
\text { me ha apoyado mucho aquí en el hospital (Entrevista 4) }\end{array}$ & \multirow{3}{*}{$\begin{array}{l}\text { Acompañar a } \\
\text { citas }\end{array}$} \\
\hline $\begin{array}{l}\text { P: El apoyo fue de... mis hijos sobre todo, mi mamá, mis hermanos estuvieron } \\
\text { todo el tiempo al pendiente de hecho de repente no te lo esperas tanto, piensas } \\
\text { que no te van a apoyar tanto como te apoyan, están al pendiente de... de cuándo } \\
\text { tienes tu cita, de acompañarte de cuidarte (Entrevista 7) }\end{array}$ & \\
\hline $\begin{array}{l}\text { P: Y ahí andábamos hasta que dimos (la paciente y su pareja no saben leer) y por } \\
\text { eso mi comadre dijo "Yo los acompaño" (Entrevista 5) }\end{array}$ & \\
\hline $\begin{array}{l}\text { P: ... Tengo una hermana que ella, si le dices "¡Hazle esto!" si le dicen “¡Hazle } \\
\text { un remedio!" siempre... ella todos los remedios caseros me hace, todo lo que le } \\
\text { decían ella me hacía. (Entrevista 9) }\end{array}$ & \multirow{2}{*}{$\begin{array}{l}\text { Preparar } \\
\text { comidas o } \\
\text { remedios }\end{array}$} \\
\hline $\begin{array}{l}\text { P: ...A veces así me decía "Tía nos venimos a almorzar con uste porque si no, no } \\
\text { va a comer, ándele" y ya me llevaban (comida), mi hermana antes de irse, ya me } \\
\text { dejaba, me daba, hasta la fecha, me daba un licuado de leche con almendras, } \\
\text { pasas y nueces. (Entrevista } 8 \text { ) }\end{array}$ & \\
\hline $\begin{array}{l}\text { P: o hay veces que, que como cuando ella se iba y me cuidaba, y los } 8 \text { días o } 15 \\
\text { días que yo me sentía más mal, ella se quedaba y me andaba dando de comer } \\
\text { "No, tienes que comer, eso te va a hacer más mal, aunque sea poquito, ándale" y } \\
\text { ella pobre, hasta con cuchara me andaba dando. (Entrevista 8) }\end{array}$ & $\begin{array}{l}\text { Cuidados } \\
\text { de la } \\
\text { enfermedad }\end{array}$ \\
\hline $\begin{array}{l}\text { P: ... Es mucha ayuda la de mi hija que está casada que hace lo posible por ella } \\
\text { traerme dos días a la semana, si ella vive en Aguascalientes y se traslada a Yahua- } \\
\text { lica y de ahí ella me, me trae otro día (Entrevista 6) }\end{array}$ & \multirow[t]{2}{*}{ Transporte } \\
\hline $\begin{array}{l}\text { P.... me dicen, "Hija, si no puede tu marido, yo voy por ti", o "que te, te suba al } \\
\text { camión y yo, yo... paso. (Entrevista } 2 \text { ) }\end{array}$ & \\
\hline
\end{tabular}


5 pacientes que contaban con un trabajo remunerado fuera de casa, lo abandonaron, debido a sintomatología (principalmente efectos secundarios de la quimioterapia), precauciones y/o recomendaciones para el cuidado de su enfermedad o al iniciar tratamientos como la quimioterapia o radioterapia.
Esta situación, refieren las pacientes, llevó a una modificación en los roles de personas cercanas, mayoritariamente de la familia nuclear, es decir, esposo e hijos. La tabla 4 muestra de manera visual aquellas personas, quienes adoptaron algún cambio de rol tras el diagnóstico de cáncer de la entrevistada.

\section{Tabla 4. Visualizador de Matriz de Códigos para Modificación de Roles}

\begin{tabular}{|c|c|c|c|c|c|c|c|c|c|c|}
\hline & $\begin{array}{c}\text { Número de } \\
\text { Entrevista }\end{array}$ & \multirow{2}{*}{1} & \multirow{2}{*}{2} & \multirow{2}{*}{3} & \multirow{2}{*}{4} & \multirow{2}{*}{5} & \multirow{2}{*}{6} & \multirow{2}{*}{7} & \multirow{2}{*}{8} & \multirow{2}{*}{9} \\
\hline $\begin{array}{c}\text { Modificación } \\
\text { de Roles }\end{array}$ & & & & & & & & & & \\
\hline \multicolumn{2}{|c|}{ En la paciente } & घ & घ & घ & घ & घ & घ & घ & - & घ \\
\hline \multicolumn{2}{|c|}{ En esposo } & 口 & 更 & 口 & & ! & & & & $\square$ \\
\hline \multicolumn{2}{|c|}{ En hijos } & & $\square$ & 口 & & 口 & 匹 & 口 & & 口 \\
\hline \multicolumn{2}{|c|}{ En hermanos } & & & & & & & घ & - & \\
\hline \multicolumn{2}{|c|}{ En otros } & & a & & & च & च & & & \\
\hline
\end{tabular}

\section{Tabla 5. Rol de Amas de Casa}

\begin{tabular}{|l|c|}
\hline \multicolumn{1}{|c|}{ Ejemplos de Narraciones } & Código \\
\hline $\begin{array}{l}\text { P: Sigo haciendo mis actividades, les digo, no me hagan sentir este... una inútil, } \\
\text { se puede decir. Y trato de darle, de... así mi vida normal (Entrevista 2) }\end{array}$ & $\begin{array}{c}\text { Sensación de } \\
\text { utilidad }\end{array}$ \\
\hline $\begin{array}{l}\text { P.... ¡No, no porque yo vivo sola con mi esposo y con mi niño y no, eso lo hago } \\
\text { yo sola! Incluso cuando me siento mal, que me han dicho que no puedo arri- } \\
\text { marme e.. a la estufa, de todos modos tengo que hacerlo todo yo porque tengo } \\
\text { que atender a mi niño... (entrevista 4) }\end{array}$ & $\begin{array}{c}\text { Responsabilidad } \\
\text { de Madre }\end{array}$ \\
\hline $\begin{array}{l}\text { P.... Pues yo a veces me arrimo, porque siempre me da lástima con mis hom- } \\
\text { bres, pos ellos son los que hacen, porque yo no tengo hijas, por nomás mi } \\
\text { esposo y mi hijo, pos a veces los veo ya medios aburridos de tanta cocina } \\
\text { (Entrevista 5) }\end{array}$ & $\begin{array}{c}\text { Ideología } \\
\text { tradicional de } \\
\text { género }\end{array}$ \\
\hline $\begin{array}{l}\text { P: ¡No, Si! Si como ahora que vengo a mis... tratamientos, pues yo tengo que } \\
\text { dejar a mis hijas en la casa, yo tengo que dejar quehacer y todo porque ps } \\
\text { siempre uno de mamá se encarga de todo ¿no? Yo sigo en mi trabajo de la casa, } \\
\text { hago todo lo que... por eso digo que yo lo tomé así como cualquier otra enfer- } \\
\text { medad. (Entrevista 6) }\end{array}$ & $\begin{array}{c}\text { Responsabilidad } \\
\text { de Madre }\end{array}$ \\
\hline $\begin{array}{l}\text { P.... ¡No!... nada más este... los primeros días de la quimio, un día, dos días, } \\
\text { tres máximo y aunque me sintiera mal yo trataba de seguir mis actividades y } \\
\text { eso me hacía sentir mejor... entonces haz de cuenta que yo me tomaba en un } \\
\text { juguito, dos, tres, cuatro, los que se pudiera y yo sentía que me lo tomaba y que } \\
\text { me levantaba y yo seguía con mis quehaceres, rápido tomaba y me cocía una } \\
\text { avena o... (entrevista 7) }\end{array}$ & $\begin{array}{r}\text { Responsabilidad } \\
\text { de Madre }\end{array}$ \\
\end{tabular}


En las tablas 2 y 4 que incluyen a la familia nuclear de las pacientes, destaca la coincidencia de otorgamiento de apoyo instrumental por parte de los hijos y el esposo, con una mayor modificación en sus actividades cotidianas y roles sociales tras el diagnóstico de CM.

Por último, la mitad de las entrevistadas destacó la dificultad para abandonar actividades tareas de hogar o el cuidado de los hijos, debido principalmente a tres razones: la responsabilidad de madre, la sensación de utilidad y una ideología tradicional de género (Ver tabla 5).

\section{DISCUSIÓN}

El impacto del cáncer en la mujer tiene consecuencias únicas, debido al papel central que ellas asumen en la vida familiar. A través de los años ha prevalecido la construcción social del rol de ama de casa de las mujeres, suelen ser ellas las responsables del manejo de la casa, la crianza de los hijos y el cuidado de la familia. Cuando se enfrentan al cáncer, las mujeres pueden ser restringidas en sus capacidades para llevar a cabo las funciones dentro de la familia como consecuencia de la enfermedad y su tratamiento, lo que lleva a los otros miembros a enfrentar un cambio de roles y responsabilidades como ajuste ante la situación de enfermedad ${ }^{(23)}$. Es la familia nuclear, quien compensa con mayor frecuencia el descuido o abandono de ciertas actividades de la paciente en el hogar, o quienes deben adoptar nuevos roles debido al desajuste económico tras el abandono del empleo de la paciente. Los movimientos funcionales, afectivos $y$ relacionales que ocurren en la familia ante un diagnóstico de CM parece ser un hecho frecuente y de gran trascendencia, que ha sido poco estudiado en nuestra población.

En el caso del grupo de mujeres entrevistadas, observamos que manifiestan en su discurso un cambio de vida a par- tir del diagnóstico, pero ese cambio no es descrito de la misma manera en cada una de ellas, y lleva implícito un énfasis en tres aspectos básicos: 1) la modificación de sus actividades cotidianas sucede a raíz del impacto de la enfermedad y la disminución de su funcionalidad tras los efectos secundarios de los tratamientos, 2) la presencia de sentimientos de inutilidad se vinculan principalmente con la idea de que han faltado a su responsabilidad como madres, sobre todo cuando aún hay niños pequeños; y así como con las recomendaciones o a cuidados acentuados que se desean instaurar tras su padecimiento; y 3) el apoyo instrumental que reciben impacta a la familia que habrá que presentar una modificación importante de roles, sobre el esposo y/o los hijos que deben asumir actividades consideradas típicamente asociadas al rol de ama de casa.

Estos aspectos deben ser considerados como un elemento importante en la comprensión y el soporte que se debe otorgar a las mujeres con $\mathrm{CM}$ y a sus familias. El tratamiento integral implicaría la asistencia para que las pacientes y sus familiares puedan transitar con el menor sufrimiento posible por el proceso de la enfermedad

En nuestros resultados hay coincidencias con lo reportado en otras investigaciones, el marido es la principal fuente de apoyo en la paciente con $\mathrm{CM}^{(19,21,23)}$, y el apoyo instrumental es el tipo de apoyo que se le otorga con más frecuencia ${ }^{(25)}$.

Notamos diferencias entre la frecuencia en la fuente de apoyo instrumental y la modificación de roles en personas específicas, según lo referido por las pacientes, esto se explica por el tipo de apoyo instrumental que brinda cada uno. De acuerdo a las narraciones de las entrevistadas, el apoyo instrumental por parte de hermanos, padres, familia extensa e incluso vecinos o amigos, con sus excepciones, estuvo más orientada a brindar cosas específicas como ayuda económica, comida, incluso aloja- 
miento o transporte temporal, por lo que sus actividades cotidianas se veían menos alteradas que el de sus parejas y/o hijos.

Aquellas pacientes que mostraron preocupación por mantener su rutina diaria, favorecían el control de sentimientos negativos, generando una sensación de bienestar; ellas mismas, buscaban estrategias para seguir desempeñando su rol de manera normal, a pesar de algunos síntomas derivados del tratamiento ${ }^{(29)}$. Afortunadamente gran parte de pacientes con $\mathrm{CM}$ no llegan a un punto incapacitante ${ }^{(16)}$. Hasta el momento de las entrevistas ninguna de las participantes se encontraba en un estado de dependencia total.

Bajo otra perspectiva, varias mujeres tienen dificultades para asumir el rol de enfermo y pedir ayuda, sobre todo aquellas que tienen hijos pequeños, a quienes les resulta más complicado prescindir de sus actividades dentro del hogar ${ }^{(30)}$. Quienes tienen hijos mayores, encuentran más apoyo en éste sentido, sin embargo, sobre todo meses después del diagnóstico, de acuerdo a lo narrado por algunas pacientes, se podría observar el desgaste que generan en ellos la nueva asignación de roles (Referirse a Tabla 5, entrevista 5), lo que pudiera hacer que la paciente retome responsabilidades. El siguiente fragmento ilustra este punto:

“... una vez que pasa e... digamos... que tu empiezas a notar que ya empiezan a cansarte de tanto chiquearte, te, te... y uno empieza como que " ¡Ay canijo, ya, ya enfadé!" a lo mejor este, si tenía que Ilegar a, a... un fin la enfermedad $y$, y ya o te... ¡O te alivias o te mueres! Y, y, es donde empieza como lo más duro ¿no? Después de, de unos meses" (Entrevista 7)

Por último, en nuestras pacientes al igual que lo referido por otros estudios ${ }^{(12-13)}$, quienes contaban con un empleo, lo aban- donaron voluntariamente al comenzar su tratamiento, centrándose en la búsqueda de la recuperación de su salud, confiando en que ésta decisión era la mejor, al disminuir el riesgo de contagio o "daño a la salud" que sus actividades o la exposición en su trabajo podría causar, o por el impedimento que los síntomas derivados de los tratamientos provoca. Este suceso puede repercutir directamente en la economía familiar.

\section{CONCLUSIÓN}

Las pacientes modificaron sus actividades cotidianas tras el diagnóstico de CM. A pesar de que un amplio número de miembros de la familia podrían brindar apoyo instrumental a las pacientes; como hermanos, padres o familia extensa, fue el esposo y los hijos quienes además de ser una fuente importante de apoyo debían, con mayor frecuencia, modificar sus actividades o adoptaban nuevos roles tras el diagnóstico de CM en la mujer.

Las amas de casa quienes refirieron resistirse o les resultaba complicado abandonar del todo actividades en el hogar, incluyendo el cuidado de los hijos, básicamente a una ideología tradicional de género.

\section{REFERENCIAS BIBLIOGRÁFICAS}

1. Anderson BO, Cazap E. Breast health global initiative (BHGl) outline for program development in Latin America. Salud Pública Mex 2009;51(Suppl 2):309-15. Doi: 10.1590/S0036-36342009000800022

2. Knaul FM, Bustreo F, Ha E, Langer A. Breast Cancer: Why link early detection to reproductive health interventions in developing countries? Salud Pública Mex 2009; 51:220-7. Doi: 10.1590/S003636342009000800012

3. Valencia-Mendoza A, Sánchez-González G, Bautista-Arredondo S, Torres-Mejia G, Bertozzi SM. Costo-Efectividad de 
políticas para el tamizaje de cáncer de mama en México. Salud Publica de Mex 2009;51:296-304. Doi: 10.1590/S003636342009000800020

4. Knaul FM, Nigeda G, Lozano R, Arreola-Ornelas H, Langer A, Frenk J. Cáncer de Mama en México: Una prioridad apremiante. Salud Pública de Mex 2009;51:335-44. Doi: 10.1590/S003636342009000800026

5. Sosa-Rubí S, Walker D, Serván E. Práctica de mastografías y pruebas de Papanicolaou entre mujeres de áreas rurales de México. Salud Pública de Mex 2009;51:236-45.

6. Riesgo Estatal de Cáncer. Guadalajara. Secretaria de Salud Jalisco.2008. [Acceso 10 de septiembre de 2013] Disponible en: http: \\ssj.jalisco.gob.mx.

7. Secoli SR, Pezo-Silva MC, Alves Rolim M, Machado AL. El cuidado de la persona con cáncer. Index Enferm 2005:34-9.

8. Ahmed RL, Prizment A, Lazovich D, Schmitz KH, Folsom AR. Lymphedema and quality of life in breast cancer survivors: the lowa Women's Health Study. J Clin Oncol 2008;10;26:5689-96. Doi: 10.1200/JCO.2008.16.4731

9. Dutta V. Chemotherapy, neurotoxicity, and cognitive changes in breast cancer. J Cancer Res Ther 2011;7:264-9. Doi: 10.4103/0973-1482.87008

10. Fu MR, Constance M, Chen J, Amber AG, Deborah A. The effect of Providing Information about Lymphedema on the Cognitive and Symptom Outcomes of Breast Cancer Survivors. Ann Surg Oncol 2010;17:184753. Doi: 10.1245/s10434-010-0941-3

11. Fu MR, Rosedale M. Breast cancer survivors' experiences of lymphedema-related symptoms. J Pain Symptom Manage. 2009;38:849-59. Doi: 10.1016/j.jpainsymman.2009.04.030

12. Mujahid MS, Janz NK, Hawley ST, Griggs JJ, Hamilton AS, Katz SJ. The impact of sociodemographic, treatment, and work support on missed work after breast cancer diagnosis. Breast Cancer Res Treat
2010;119:213-20. Doi: 10.1007/s10549009-0389-y

13. Font A, Rodríguez E. Eficacia de la terapia de grupo en cáncer de mama: variaciones en la calidad de vida. Psicooncología 2004;1:67-86.

14. Salas C, Grisales H. Calidad de vida y factores asociados en mujeres con cáncer de mama en Antioquia Colombia. Rev Panam Salud Pública 2010;28:9-18.

15. Rojas-May G. Estrategias de intervención psicológica en pacientes con cáncer de mama. Rev Med Clin Condes 2006; 17:194-7.

16. López Cuevas Z, González Ortega J, Gómez Hernández M, Morales Wong M. Impacto psicológico y social de la mastectomía en las pacientes operadas de cáncer de mama. Rev Med Electrón [seriada en línea] 2008:30(2) [Acceso 13 de septiembre de 2013]. Disponible en: http://www. revmatanzas.sld.cu/revista\%20medica/ ano\%202008/vol2\%202008/tema01.htm

17. Robles R, Morales M, Jiménez L, Morales J. Depresión y ansiedad en mujeres con cáncer de mama: el papel de la efectividad y el soporte social. Psicooncología 2009;6:191-201.

18. Londoño YC. El proceso de adaptación en las mujeres con cáncer de mama. Inves Educ Enferm 2009;XXVII:70-7.

19. Carrión C, Galdón A, Manzanares A, Rodenas A, Rodríguez M. Aspectos psicosociales del cáncer de mama. Rev Enferm 2004;18:19-23.

20. Gamarra CJ, Paz EP, Griep RH. Social support and cervical and breast cancer screening in Argentinean women from a rural population. Public Health Nurs 2009;26:269-76. Doi: 10.1111/j.15251446.2009.00779.x

21. Hasson-Ohayon I, Goldzweig G, Braun M, Galinsky D. Women with advanced breast cancer and their spouses: diversity of support and psychological distress. Psychooncology 2010;19:1195-204. Doi: 10.1002/pon.1678 
22. Malya R, Umezawab Y, Leakec B, Silliman R. Mental health outcomes in older women with breast cancer: impact of perceived family support and adjustment. Psychooncology 2005;14:535-45. Doi: 10.1002/pon.869

23. Mokuau N, Braun KL. Family support for Native Hawaiian women with breast cancer. J Cancer Educ 2007;22:191-6. Doi: 10.1007/BF03174336

24. Arora NK, Finney Rutten LJ, Gustafson DH, Moser R, Hawkins RP. Perceived helpfulness and impact of social support provided by family, friends, and health care providers to women newly diagnosed with breast cancer. Psychooncology 2007;16:474-86. Doi: 10.1002/ pon. 1084

25. Baider L. Cáncer y familia: aspectos teóricos y terapéuticos. Rev Inter Psicol Clín Salud 2003;3:505-20.
26. Goetz JP, LeCompte MD. Características y orígenes de la etnografía educativa. Etnografía y diseño cualitativo en investigación educativa. Madrid: Morata, 1988.

27. Vela Peón F. Un acto metodológico básico de la investigación social: la entrevista cualitativa. In: Sociales LC, editor. Observar, escuchar, comprender la tradición Cualitativa en la investigación social, 2001. p. 63-95.

28. Kornblit A. Metodologías Cualitativas: Modelos y Procedimientos de Análisis. $2^{\circ} \mathrm{ed}$. Buenos Aires Biblios, 2007.

29. Palmadottir G. The role of occupational participation and environment among Icelandic women with breast cancer: A qualitative study. Scand J Occup Ther 2010;17:299307. Doi: 10.3109/11038120903302874

30. Coyne E, Borbasi S. Living the experience of breast cancer treatment: The younger women's perspective. Aust J Adv Nurs 2008;26:6-13. 
